\title{
Roll Motion Analysis of A High Speed Mono-hull Ship
}

\author{
Zhiwei CAO ${ }^{1, a, *}$, Shasha GAO $^{2, b}$, Songlin $\mathrm{YANG}^{3, \mathrm{c}}$ \\ ${ }^{1,2,3}$ School of Naval Architecture \& Ocean Engineering, Jiangsu University of Science and Technology, \\ Zhenjiang, Jiangsu, China
}

897118610@qq.com ${ }^{\mathrm{a}}$,1679488425@qq.com ${ }^{\mathrm{b}}$,ysl560516@vip.163.com

Keywords: High speed mono-hull ship, Roll motion model, System identification, Genetic algorithm

\begin{abstract}
In this paper, a high speed mono-hull ship is taken as the research object, and the roll decay tests are carried out under different drafts and different initial rolling angles of two ship models with or without bulbous bow, The movement process of the roll motion of a high speed mono-hull ship is studied by rolling test. A mathematical model of system identification for roll motion of high speed mono-hull ship based on genetic algorithm (GA) is established, and a calculation program is compiled and a practical example of the roll of experimental data is calculated. Compared the experimental results and the identification results, two reasonable mathematical models of roll motion are obtained, which provides a basis for further study on high speed mono-hull ship performance, meanwhile verifies the system's reliability.
\end{abstract}

\section{Introduction}

When the ship is sailing on the sea, the environment is complex and changeable, and it is the key to ensure the smooth movement of the ship and the normal operation of the electronic equipment on board. The ship rolling motion has great influence on the electronic equipment, so the study of ship rolling motion is essential to the performance.

Dengcheng $\mathrm{Liu}^{[1]}$ used analytic method to calculate the natural period and dimensionless attenuation coefficient of ship model, and the calculation process was complex and difficult to distinguish the coefficient reliability. Lin Wang and Ping $\mathrm{Ma}^{[2]}$ fully introduced the system identification method, and analyzed the shortcomings of traditional identification methods. They put forward some new identification methods using neural networks, genetic algorithms and other knowledge for system identification. Wanglin Yang ${ }^{[3]}$ identified the rolling experimental data of an unmanned planing boat under different initial conditions, and verified the reliability of the identification method. Xuequan $\mathrm{Ma}^{[4]}$ used the identification software based on fuzzy genetic algorithm (GA) to perform roll identification simulation for a LPG ship. It is verified that the method of system identification in the field of reliability analysis in seakeeping experiment. The system identification method has also been applied in the rolling motion experimental analysis of the trimaran model, the five-body model and the composite boat ${ }^{[5-8]}$.

In this paper, the roll motion of a high speed mono-hull ship model is experimented and analyzed. Considering the influence of the linear and nonlinear factors of the roll of high speed mono-hull ship, three mathematical models of system identification for high-speed haulage based on genetic algorithm established. A part of experimental data imported into the self compiled identification program and the equations of ship roll motion obtained. The identification data are taken into the equation, and the calculated curve is fitted with the experimental curve. The reliability of the identification program is verified by the better fitting and it has less error of fitting.

\section{Experimental Model and experimental Scheme}

\subsection{Model Introduction}

In this paper, a high speed mono-hull ship is chosen as the object of study. Designed and 
installed a bulbous bow to the prototype ship, and produced a scale model with ball-free bow 2.63 meters and a spherical bulb of 2.73 meters. The main dimensions of a prototype ship model are shown in table 2.1.

Table 2.1 The main scale parameters of prototype ship

\begin{tabular}{|c|c|c|c|c|}
\hline No. & Design variable name & Symbol & Unit & Size \\
\hline 1 & Length & $\mathrm{L}$ & $\mathrm{m}$ & 2.63 \\
\hline 2 & Width & $\mathrm{B}$ & $\mathrm{m}$ & 0.23 \\
\hline 3 & Moulded depth & $\mathrm{T} 1$ & $\mathrm{~m}$ & 0.14 \\
\hline 4 & Designed draft & $\mathrm{T} 2$ & $\mathrm{~m}$ & 0.083 \\
\hline 5 & Mid-ship section coefficient & $\mathrm{Cm}$ & & 0.877 \\
\hline 6 & Block coefficient & $\mathrm{Cb}$ & & 0.536 \\
\hline
\end{tabular}

Table 2.2 The bulbous bow model parameters

\begin{tabular}{|c|c|c|c|c|}
\hline No. & Design variable name & Symbol & Unit & Size \\
\hline 1 & Relative length of protrusion & $\mathrm{lb} / \mathrm{lbp}$ & & 0.044 \\
\hline 2 & Relative submergence & $\mathrm{hb} / \mathrm{T}$ & & 0.614 \\
\hline 3 & Maximum width ratio & $\mathrm{bmax} / \mathrm{B}$ & & 0.212 \\
\hline 4 & Relative displacement ratio & $\delta / \Delta$ & & 0.028 \\
\hline
\end{tabular}

Figure 2.1 The original ship model and the Bulbous bow

\subsection{Experimental Equipment}

MTi-G is a miniature inertial measurement system with integrated GPS signals. It can output triaxial acceleration, triaxial angular velocity, triaxial attitude angle (pitch / roll / yaw). Built-in high-precision anti-vibration gyroscope chip, and it can high-precision output triaxial attitude angle in vibrating environment and non-uniform magnetic field environment.

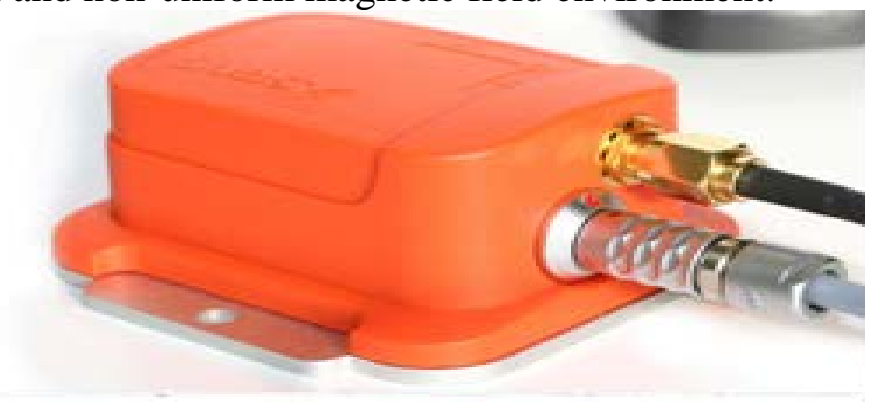

Figure 2.2 MTi-G miniature AHRS inertial measurement system

The Mti will be placed at the center of gravity of the ship model. After the ship is launched,we can adjust the ballast so that the ship model is floating.Giving the ship an initial roll angle and then letting it sway, passing the data measured by Mti and saving it to on the shore of the computer.

\subsection{Experiment Content}

The experimental procedure of this experimental is as follows:

1)The ship model placed in the pool, to be stable after the ship model, adjust the ballast position 
so that the model is in upright condition, read its initial angle in the still water;

2)Under standard draft state, the external force is applied to the ship model so that its initial roll angle is $5,7,9,12$, and 15 degrees. While releasing the external force, the Mti-G software is used to collect the data. Until the ship model shakes steady, it ends to collection of data and the roll angle data can be obtained;

3)Increase the ballast load by $5 \%$ standard drainage, repeat steps (2);

4)Continue to increase the standard volume of $5 \%$ of the ballast, repeat step (2);

5)After the ship model without bulbous bow experimental well done, the boat model should be carried to shore. And then the bulbous bow will be placed in the water, repeat step(1) (4).

\section{System Identification}

A large number of literature shows that system identification has been widely used in ship model experimental analysis. It is good in identifying ship maneuverability, wave resistance hydrodynamic derivative, and establishing mathematical model.This article uses a self-made system identification program based on genetic algorithm ${ }^{[9-10]}$.

\subsection{Genetic Algorithm}

The concept of genetic algorithm (GA) was first proposed by Professor John Holland of the United States in the monograph Adaptation in Nature and Artificial System. Just as its name implies, this concept mimics the evolutionary processes and mechanisms of the creatures. The algorithm randomly searches the computational objects by simulating the principles of selection and elimination of natural biology and genetic laws.

The basic elements of genetic algorithm are mainly including: population, fitness function, selection strategy, genetic strategy and convergence criterion. Among them, genetic strategies include crossover and mutation.

The operation steps of the genetic algorithm are as follows:

(1)Initial population

The initial population was randomly generated by 0-1 encoding. Each individual corresponds to a chromosome. Which is equivalent to a solution of the corresponding problem, the length of chromosomes fixed length of 0,1 strings. The population size is usually set at 100 to 1000 , the initial population set in this paper is 200 . In general, the initial population size and genetic algebra should be taken into account in order to obtain optimal results.

Individual fitness function

To determine the fitness function of each individual in the population, the calculated individual fitness value is an important basis for selection in the evolutionary process. The fitness function is usually designed according to the objective function. The final determination of the fitness function should be analyzed in detail. For example, some fitness functions can directly select the objective function, and some can transform the objective function into linear or transform the power function in order to be used as fitness function. Of course, the fitness function should be chosen properly, otherwise it may affect the optimization performance of the algorithm.

(3)Genetic strategy

Genetic strategy is divided into crossover and mutation, which is the essence of genetic algorithm. Crossover is the intersection of two genes of two current individuals, resulting in two new individuals. The commonly used intersection methods include single point crossing, double point crossing, uniform crossing, and so on. Variation is the formation of new individuals on chromosomes by selecting a number of alleles and replacing them with other alleles. The crossover probability chosen in this paper is 0.8 and the probability of mutation is 0.15 .

(4)Selection strategy

Selection refers to the selection of good individuals in the current population with certain probability, thus generating the next generation of the mating pool.By choosing, we select the intermediate solution which is close to the optimal solution. The fitness value is the selection principle. Individuals with high fitness values are selected with greater probability, and individuals 
with low fitness values are less likely to be selected. The common selection strategies are roulette, random competition, random league, local selection and so on.

(5)Convergence criteria

The maximum iteration number is reached and the algorithm terminates. If the fitness and fitness of the optimal individuals are not stable, the population should be selected, crossed and altered, and the fitness value should be re calculated until the convergence judged.

\subsection{System Identification}

System identification is based on the measured input and output data of the actual system.According to the specific identification criteria, from a given set of model classes to determine a property that reflects the actual system itself ${ }^{[11]}$.

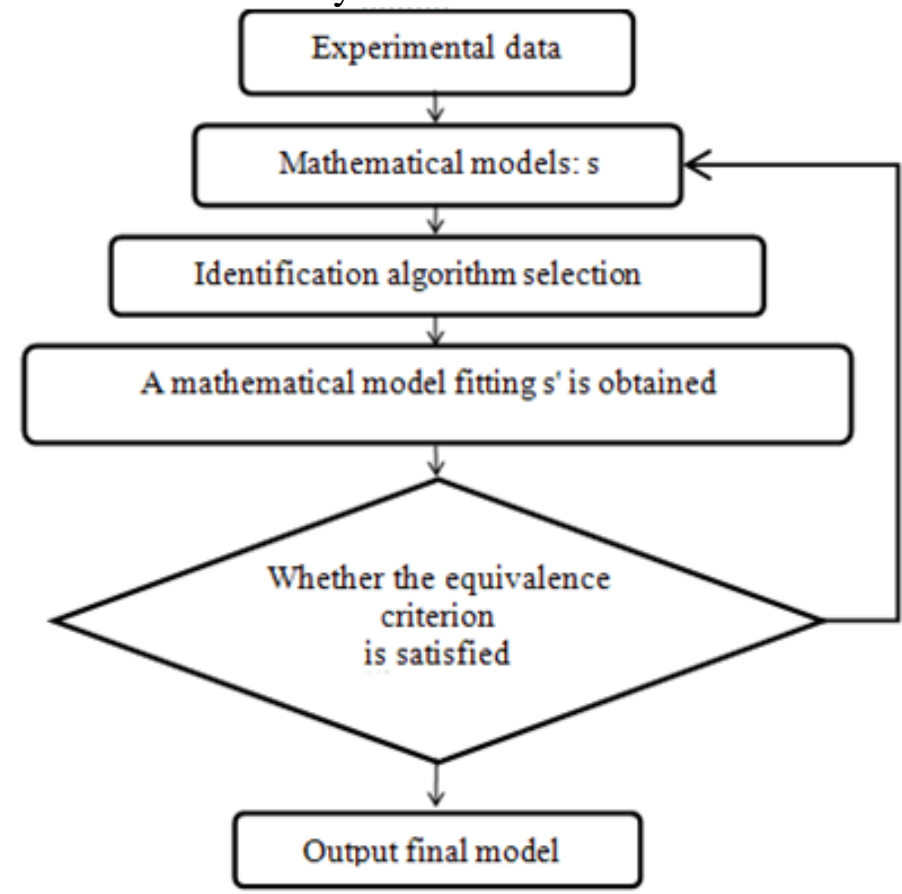

Figure 3.1 System identification flow chart

\subsection{Identification mathematical model}

Three mathematical models are adopted in this paper, which take linear and nonlinear factors into account ${ }^{[5-8]}$, model $1,2,3$ respectively,

1)The model 1 is when the damping angle and the restoring moment are linear, the model equation is:

$$
\ddot{\varphi}+2 \frac{N_{\theta}}{I_{X}} \dot{\varphi}+\frac{D h}{I_{X}} \sin \varphi=0
$$

2)The model two is when the damping moment is nonlinear and the recovery moment is linear, the model equation is:

$$
\ddot{\varphi}+2 \frac{N_{\theta}}{I_{X}} \dot{\varphi}+\frac{W}{I_{X}}|\dot{\varphi}| \dot{\varphi}+\frac{x}{I_{X}} \dot{\varphi}^{3}+\frac{D h}{I_{X}} \sin \varphi=0
$$

3)The model three is that when the damping moment and the restoring moment are nonlinear, the model equation is:

$$
\ddot{\varphi}+2 \frac{N_{\theta}}{I_{X}} \dot{\varphi}+\frac{W}{I_{X}}|\dot{\varphi}| \dot{\varphi}+\frac{x}{I_{X}} \dot{\varphi}^{3}+\frac{C_{1}}{I_{X}} \varphi^{3}+\frac{C_{2}}{I_{X}} \varphi^{2}+\frac{C_{3}}{I_{X}} \varphi=0
$$

Where: $\mathrm{h}$-high initial stability of high speed mono-hull ship; $D$ - the displacement of high 
speed single ship; $I_{x}$ — total moment of inertia of hull roll; $\varphi$-roll angle; $\dot{\varphi}$-roll angular velocity; $\ddot{\varphi}$-roll angular acceleration; $N_{\theta}$-coefficient of roll type damping moment; $C_{1}, C_{2}$-nonlinear restoring moment coefficient; $C_{3}$-Linear restoring moment coefficient.

Prediction of roll angular velocity at $\mathrm{K}+1$ moments (model 1 ):

$$
\dot{\varphi}_{K+1}=\left(-2 \frac{N_{\theta} t}{I_{X}}+1\right) \dot{\varphi}_{K}-\left(\frac{D h}{I_{X}} \sin \varphi\right) t
$$

By measuring the angle at $\mathrm{k}$ moment, there is an error estimation criterion at time $\mathrm{k}+1$ :

$$
\varepsilon_{k+1}=\dot{\varphi}_{k+1}^{\prime}-\dot{\varphi}_{k+1}
$$

$\dot{\varphi}^{\prime}{ }_{K+1}$ - the measured value of angular velocity at $\mathrm{k}+1$ moment;

-

$\varphi_{K+1}$-identification result.

The loss function can be expressed as:

$$
J_{k+1}=\left|\varepsilon_{k+1}\right|
$$

Then select the target function as:

$$
F(x)=\sum_{k=1}^{N} J_{k+1}^{2}
$$

Take $I_{x}, N_{\varphi}, W, x, C_{1}, C_{2}, C_{3}$ as design variables, according to experience, the upper and lower bounds of $I_{x}$ are $(0,0.2)$; the upper and lower bounds of $\mathrm{W}$ are $(0,1)$; the upper and lower bounds of $N_{\varphi}$ are $(0,1)$; the upper and lower bounds of $\mathrm{x}$ are $(0,10)$; the upper and lower bounds of $\mathrm{C}_{1}$ are $(0,5)$; the upper and lower bounds of $\mathrm{C}_{2}$ are $(5,10)$; the upper and lower bounds of $\mathrm{C}_{3}$ are $(0,10)$. Design variables, constraints, and objective functions constitute a standard optimization mathematical model.

The initial population number was 200, the number of iterations was 3000 , the crossover rate was 0.8 , and the mutation rate was 0.15 . Adopt growth mechanism (inner carrier) mode.

\section{Experimental result analysis}

\subsection{Roll motion under the same initial roll angle and draft}

Comparing two sets of angular velocity data of the two models in the designed draft and the initial roll angle of $5^{\circ}$, results are as follows:

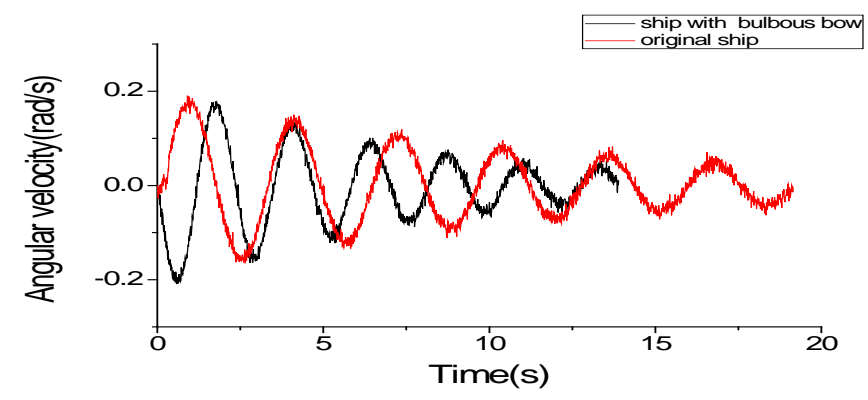

Figure 4.1 The decay curve of rolling angular velocity in designed draft

Select and analysis the first six cycles of the roll experimental, we can find that the rolling period of a ship with a bulbous bow is short,and angular velocity decays faster.This picture shows that the bulbous bow has the effect of reducing roll. 


\subsection{Identification result analysis}

Identification data selection rule: select the first six cycles of the roll,each cycle is divided into 24 sub-intervals, identify the data from the two-thirds key sections.

1) Select the ship model without a bulbous bow under standard draft, and identify the condition of the initial roll angle is five-degree, then the roll motion equation under this condition is obtained by identification:

$$
\ddot{\varphi}+0.3851 \dot{\varphi}+129.758 \sin \varphi=0
$$

Changing the mathematical model of identification, the roll motion equations are established when the restoring moment is linear, the damping moment is nonlinear and the restoring moment and damping moment are nonlinear:

$$
\begin{array}{r}
\ddot{\varphi}+0.383 \dot{\varphi}+0.053|\dot{\varphi}| \dot{\varphi}+0.154 \dot{\varphi}^{3}+129.256 \sin \varphi=0 \\
\ddot{\varphi}+0.304 \dot{\varphi}+0.064|\dot{\varphi}| \dot{\varphi}+0.136 \dot{\varphi}^{3}+0.338 \varphi^{3}+25 \varphi^{2}+2.76 \varphi=0
\end{array}
$$

The experimental data are brought into the three equations of motion and the calculated curves are obtained,as shown in Figure 4.2.

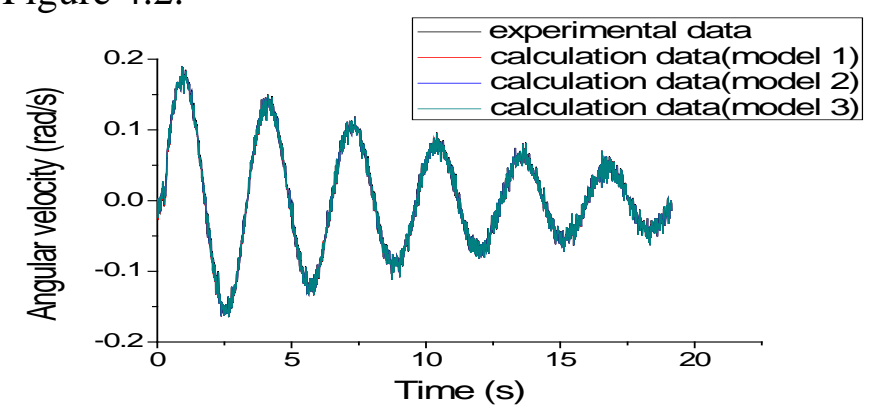

Figure 4.2 Comparison chart of experimental curve and calculation curve

Table 4.1 Error of angular velocity and calculated angular velocity

\begin{tabular}{c|c|c|c|c|c|c|c}
\hline \multicolumn{2}{c|}{} & \multicolumn{2}{c|}{ Model one } & \multicolumn{2}{c}{ Model two } & \multicolumn{2}{c}{ Model three } \\
\hline $\begin{array}{c}\text { Time } \\
(\mathrm{s})\end{array}$ & $\begin{array}{c}\text { Experimental } \\
\text { angular } \\
\text { velocity }\end{array}$ & $\begin{array}{c}\text { Calculated } \\
\text { angular } \\
\text { velocity }\end{array}$ & Error(\%) & $\begin{array}{c}\text { Calculated } \\
\text { angular velocity }\end{array}$ & Error(\%) & $\begin{array}{c}\text { Calculated } \\
\text { angular } \\
\text { velocity }\end{array}$ & Error(\%) \\
\hline 0.66 & 0.132934748 & 0.14387161 & 8.227240 & 0.143835167 & 8.199826 & 0.142311544 & 7.0536829 \\
\hline 0.67 & 0.158406083 & 0.144869662 & 8.545392 & 0.144815023 & 8.579885 & 0.142594553 & 9.9816432 \\
\hline 0.68 & 0.152978109 & 0.145808137 & 4.686927 & 0.145735366 & 4.734496 & 0.142867146 & 6.6094183 \\
\hline 0.69 & 0.149314663 & 0.146689171 & 1.758361 & 0.146598336 & 1.819196 & 0.143128492 & 4.1430431 \\
\hline 0.7 & 0.141965081 & 0.147514271 & 3.908841 & 0.147405445 & 3.832184 & 0.14337775 & 0.9950817 \\
\hline 0.71 & 0.16223359 & 0.148286236 & 8.597081 & 0.148159494 & 8.675204 & 0.143614478 & 11.476730 \\
\hline 0.73 & 0.162741481 & 0.149648739 & 8.045116 & 0.149486519 & 8.144796 & 0.144040076 & 11.491479 \\
\hline 0.74 & 0.156809107 & 0.150239558 & 4.189520 & 0.150059804 & 4.304152 & 0.144226334 & 8.0242615 \\
\hline
\end{tabular}

Random calculated missing data and fitted it, by comparing the angular velocity errors of model one and model two, we found that when the ship model rolling at a small angle, the fitting error of the two models coincide basically. It is shown that the nonlinear damping moment has little influence when the ship model rolling at a small angle.

Comparing the angular velocity errors of model two and model three, we found that when the nonlinear restoring moment is added, the error of data fitting increased. It is shown that the mathematical model with linear restoring moment is more close to reality when the ship model rolling at a small angle.

By comparing the objective function of three models, the above conclusions can be verified. 
2) Select the ship model with a bulbous bow under standard draft ,and identify the condition of the initial roll angle is five-degree, then the roll motion equation under this condition is obtained by identification:

$$
\ddot{\varphi}+0.76 \dot{\varphi}+185.493 \sin \varphi=0
$$

Changing the mathematical model of identification, the roll motion equations are established when the restoring moment is linear, the damping moment is nonlinear and the restoring moment and damping moment are nonlinear:

$$
\begin{aligned}
& \ddot{\varphi}+0.084 \dot{\varphi}+0.038|\dot{\varphi}| \dot{\varphi}+34.534 \dot{\varphi}^{3}+185.804 \sin \varphi=0 \\
& \ddot{\varphi}+0.1 \dot{\varphi}+0.092|\dot{\varphi}| \dot{\varphi}+35.456 \dot{\varphi}^{3}+0.139 \varphi^{3}+25 \varphi^{2}+3.795 \varphi=0
\end{aligned}
$$

Table 4.2 Comparison table of objective function

\begin{tabular}{|c|c|c|}
\hline & Original ship & Ship with bulbous bow \\
\hline Mathematical model & Object function & Object function \\
\hline Model one & 0.011739314 & 0.012331788 \\
\hline Model two & 0.011739383 & 0.012257743 \\
\hline Model three & 0.011983446 & 0.012455629 \\
\hline
\end{tabular}

Comparing the objective function of three models, we can find that the mathematical model when the restoring moment is linear and the damping moment is nonlinear fit best, compared the ship model without bulbous bow, the bulbous bow increases the damping moment.

3) Select the ship model without a bulbous bow under standard draft ,and identify the condition of the initial roll angle is fifteen-degree, then the roll motion equation under this condition is obtained by identification:

$$
\ddot{\varphi}+0.538 \dot{\varphi}+0.06|\dot{\varphi}| \dot{\varphi}+1.307 \dot{\varphi}^{3}+135.687 \sin \varphi=0
$$

Change the mathematical model of identification, We take the same data into the identification program,the roll motion equations are established:

$$
\ddot{\varphi}+0.525 \dot{\varphi}+0.0023|\dot{\varphi}| \dot{\varphi}+0.323 \dot{\varphi}^{3}+24.98 \varphi^{3}+25 \varphi^{2}+5.447 \varphi=0
$$

As the roll angle is relatively large, so the damping moment taken as a non-linear situation.

The experimental data are brought into the two equations of motion and the calculated curves are obtained,as shown in Figure 4.3.

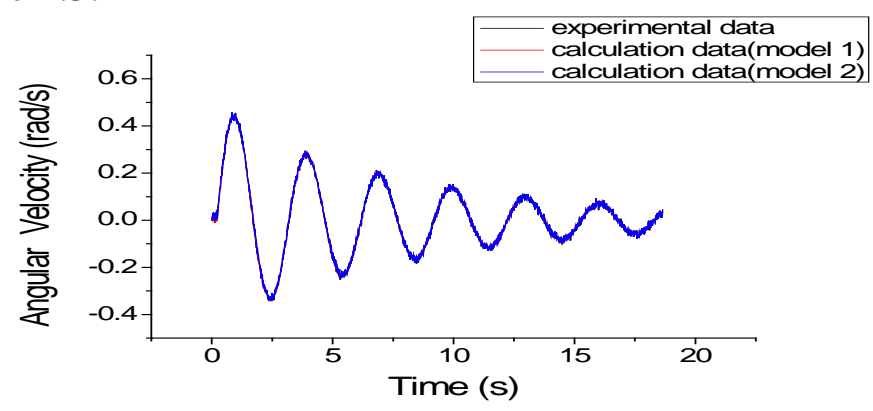

Figure 4.3 Comparison chart of experimental curve and calculation curve 
Table 4.4 Error of angular velocity and calculated angular velocity

\begin{tabular}{|c|c|c|c|c|c|}
\hline & \multicolumn{2}{|c|}{ Model one } & \multicolumn{2}{c|}{ Model two } \\
\hline $\begin{array}{c}\text { Time } \\
(\mathrm{s})\end{array}$ & $\begin{array}{l}\text { Experimental } \\
\text { angular velocity }\end{array}$ & $\begin{array}{l}\text { Calculated } \\
\text { angular velocity }\end{array}$ & Error(\%) & $\begin{array}{c}\text { Calculated } \\
\text { angular velocity }\end{array}$ & Error(\%) \\
\hline 0.69 & 0.400446598 & 0.394722211 & 1.429501 & 0.383449401 & 4.244560 \\
\hline 0.71 & 0.40528116 & 0.400145411 & 1.267206 & 0.385753113 & 4.818395 \\
\hline 0.72 & 0.398683816 & 0.402591567 & 0.980163 & 0.386921889 & 2.950189 \\
\hline 0.73 & 0.401385585 & 0.404862742 & 0.866288 & 0.388096159 & 3.310887 \\
\hline 0.74 & 0.408525727 & 0.40695982 & 0.383307 & 0.389271469 & 4.713107 \\
\hline 0.75 & 0.419826734 & 0.408882095 & 2.606942 & 0.39044323 & 6.998959 \\
\hline 0.76 & 0.425268671 & 0.410627385 & 3.442832 & 0.391606494 & 7.915508 \\
\hline 0.77 & 0.432457682 & 0.412195702 & 4.685309 & 0.392755938 & 9.180492 \\
\hline 0.78 & 0.411836617 & 0.413586452 & 0.424886 & 0.393885889 & 4.358701 \\
\hline
\end{tabular}

Random calculated missing data and fitted it, by comparing the angular velocity errors of model one and model two, we found that when the ship model rolling at a large angle the mathematical model two fit best, It is shown that the nonlinear restoring moment has little influence when the ship model rolling at a large angle.

4) Select the ship model with a bulbous bow under standard draft ,and identify the condition of the initial roll angle is fifteen-degree, then the roll motion equation under this condition is obtained by identification:

$$
\ddot{\varphi}+0.679 \dot{\varphi}+0.038|\dot{\varphi}| \dot{\varphi}+0.092 \dot{\varphi}^{3}+239.065 \sin \varphi=0
$$

Change the mathematical model of identification, We take the same data into the identification program,the roll motion equations are established:

$$
\ddot{\varphi}+0.748 \dot{\varphi}+0.009|\dot{\varphi}| \dot{\varphi}+0.493 \dot{\varphi}^{3}+0.002 \varphi^{3}+25.005 \varphi^{2}+5.316 \varphi=0
$$

As the roll angle is relatively large, so the damping moment taken as a non-linear situation.

Random calculated missing data and fitted it, we found that when the hip model with a bulbous bow has a large angle roll, it is better to use mathematical model with linear restoring moment.

Table 4.5 Comparison of formula coefficients

\begin{tabular}{|c|c|c|}
\hline Coefficient formula & $\begin{array}{c}\text { The ship model without } \\
\text { bulbous bow }\end{array}$ & $\begin{array}{c}\text { The ship model with } \\
\text { bulbous bow }\end{array}$ \\
\hline $2 \frac{N_{\theta}}{I_{X}}$ & 0.537790046 & 0.678541539 \\
\hline$\frac{W}{I_{x}}$ & 0.060294576 & 0.037553262 \\
\hline$\frac{x}{I_{X}}$ & 1.306701364 & 0.092236038 \\
\hline$\frac{D h}{I_{x}}$ & 135.6869126 & 239.0649858 \\
\hline
\end{tabular}

By comparing the coefficients of rolling motion equation when the ship models rolling at fifteen-degree under the standard draft, it is known that the bulbous bow has mainly increased the linear damping moment and the restoring moment of the ship model.

\section{Conclusion}

This paper mainly studies the roll experimental of a high speed mono-hull ship with or without bulbous bow, each ship model studied 15 kinds of conditions that include three kinds of drafts, five kinds of rolling angle. Some of the experimental data is identified by using an identification program based on genetic algorithm, the mathematical model of rolling motion under different working conditions is obtained. We can know that the bulbous bow has some influence to reduce the rolling of the ship model. At the same time, the reliability of the identification program is 
verified. This program can predict the rolling state of the ship, and provide a good basis for further research on the performance of the high speed mono-hull ship.

\section{References}

[1] Liu Dengcheng, Ding Yong. Analytic method for ship nonlinear rolling in calm water [J]. Journal of Ship Mechanics 2009, 13(1) 41-46.

[2]WANG Lin, MA Ping. Review on Methods of System Identification[J].Information On Electric Power, 2001 (4):63-66

[3]Yang Wanglin, Xu Haitong, Yang Songlin. Experimental analysis of roll motion model of unmanned planing boat[J].Ship Science and Technology, 2014,36(4)

[4]Ma Xuequan, Ji Sheng, Wen Yiyan. The system identification and Simulation of ship roll motion[J].Journal of Shanghai ship and shipping research Institute, 2016, 39(2)

[5] Huilei LI,Feng ZHU,Songlin YANG.Primary Analysis on Rolling Motion Of Trimaran [A].

Procedings of 2011Asia-Pacific Youth Conference on Communication(2011APYCC)[C],

Hangzhou, 2011.

[6] Wang Yong, Yang Songlin, Fan Kai. Research on the Analysis method of Rolling motion of Pentamaran[C].Academic Conference on ship hydrodynamics and ITTC30 anniversary of Chinese shipping academic circle. Hangzhou, 2008.

[7] Cui Jian, Wen Yiyan, Yang Songlin. Preliminary research on a new type of compoun unmanned trisomy dramwing[C].The sixteenth China International Boat and its technology Equipment Exhibition and high performance Ship academic Report, Shanghai 2011.

[8] Ma Tianyu, Cui Jian, Yang Songlin, Trimaran manipulation and roll coupling exercise test analysis [J]. Ship Science and Technology, 2012, 34 (11) : 24-26, 36.

[9] Imen Cherif, Farhat Fnaiech. Nonlinear System Identification With A Real-Coded Genetic Algorithm (RCGA)[J]. International Journal of Applied Mathematics and Computer Science.

2015(4)

[10]She Minghong. Analysis and research of genetic algorithm applied in system identification [J].

Research Findings, 2016, 11.

[11] Ding Feng. System identifcation.Part A: Introduction to the identification[J].Journal of Nanjing University of Information Science \& Technology(Natural Science Edition), 2011,01:1-22. 